\title{
A COVID-19 E O DISTANCIAMENTO SOCIAL: QUANDO A ONDA DA INTERNET SUBSTITUIU A ONDA DO MAR PARA A PRÁTICA DE EXERCÍCIOS FÍSICOS
}

\author{
Roberto Miranda Ramos Costa \\ Mestre em Ciências da Reabilitação pelo Programa de Pós-graduação em Ciências da \\ Reabilitação do Centro Universitário Augusto Motta (UNISUAM), Rio de Janeiro, RJ, Brasil \\ betomrcosta@hotmail.com \\ Patrícia dos Santos Vigário \\ Doutora em Ciências pela Faculdade de Medicina da Universidade Federal do Rio de Janeiro \\ (UFRJ), Rio de Janeiro, RJ, Brasil \\ Programa de Pós-graduação em Ciências da Reabilitação do Centro Universitário Augusto Motta \\ (UNISUAM), Rio de Janeiro, RJ, Brasil \\ patriciavigario@yahoo.com.br
}

\section{RESUMO}

Uma pandemia causada pelo novo coronavírus, o Sars-CoV-2, determinou mudanças na rotina das pessoas em todo o mundo. Dentre elas, o distanciamento social, implementado pela Organização Mundial da Saúde para conter o avanço do contágio entre as pessoas. Tal medida, impactou também na prática de exercícios físicos ao ar livre, restringindo o funcionamento dos estabelecimentos. Dentro desse contexto, medidas estão sendo tomadas pelos profissionais de educação física para propiciar a prática regular de exercícios físicos utilizando recursos tecnológicos. Os objetivos do presente estudo foram: (i) investigar o impacto da interrupção da prática de natação em águas abertas (NAA) devido ao distanciamento social frente à pandemia da Covid-19 na percepção de bem-estar e estado geral de saúde, e (ii) investigar a percepção sobre a necessidade de adaptação em um curto espaço de tempo a um novo modelo de exercício físico, realizado em ambiente domiciliar, e mediado por recursos tecnológicos utilizando a internet. A interrupção da prática de NAA, devido à recomendação de distanciamento social para a diminuição do contágio da Covid-19, trouxe repercussões negativas na percepção de saúde e bem-estar, principalmente nos aspectos relacionados à qualidade do sono e ansiedade. Entretanto, as videoaulas de ginástica por meio de ferramentas tecnológicas de internet mostraram-se positivas para minimizar tais repercussões, sobretudo por permitirem um convívio social, mesmo que virtual. Dessa forma, em tempos de distanciamento social, medidas devem ser adotadas para o equilíbrio mente sã, corpo são.

Palavras-chave: Exercício físico. Natação. Distanciamento social. Pandemia. Saúde. 


\title{
COVID-19 AND SOCIAL DISTANCING: WHEN THE INTERNET WAVE REPLACED THE SEA WAVE FOR THE PRATICE OF PHYSICAL EXERCISES
}

\begin{abstract}
A pandemia caused by the new coronavirus, Sars-CoV-2, has led changes in the routine of people around the world. Among them, the social distancing, implemented by the World Health Organization to contain the spread of Covid-19 contagion. Social distancing also had an impact on the practice of physical exercises outdoors, restricting the operation of establishments. Within this context, measures are being taken by physical education professionals to provide regular physical exercise using technological resources. The objectives of the present study were: (i) to investigate the impact of the interruption of open water swimming (NAA) due to the social distance from the Covid-19 pandemia in the perception of well-being and general health status, and (ii) to investigate the perception of the need to adapt in a short period of time to a new model of physical exercise, performed in the home environment, and mediated by technological resources using the internet. The interruption of the practice of NAA due to the recommendation of social distance to reduce the contagion of Covid-19 brought negative repercussions on the perception of health and well-being, mainly in aspects related to the quality of sleep and anxiety. However, gymnastics video classes using technological internet tools proved to be positive in order to minimize such repercussions, especially because they allow social, even virtual, interaction. Thus, in time of social distancing measures must be adopted to keep mind and body helthy.
\end{abstract}

Keywords: Physical exercise. Swimming. Social distancing. Pandemia. Health. 


\section{INTRODUÇÃO}

A natação em águas abertas (NAA) é caracterizada pela a prática da natação em mares, rios, lagos, oceanos ou em canais (BALDASSARRE et al., 2019). É um esporte democrático, em que podem praticar homens e mulheres de diferentes faixas etárias, com objetivos que vão desde a manutenção da saúde e do bem-estar, ao alto rendimento. Nos últimos anos, a popularidade deste esporte cresceu de forma expressiva, sobretudo após a sua inclusão, em 2008, no Programa Olímpico. (OPEN, 2020).

Indiscutivelmente, um dos elementos que impulsiona a prática da NAA é o contato com a natureza, diferente do que acontece em piscinas - um ambiente mais "frio" e "previsível". O contato com o mar se contrapõe ao ambiente da piscina, pois as condições marítimas são influenciadas por fatores como o vento, o clima, as correntes, as marés, o tipo de areia e, por isso, fazem com que cada dia de prática seja único. (FREITAS FILHO et al., 2018).

No Brasil, o Rio de Janeiro é uma cidade que possui um extenso litoral e propicia a prática de NAA. Localizada na região sudeste, possui clima tropical atlântico, com temperatura média anual de $23^{\circ} \mathrm{C}$, podendo chegar a $40^{\circ} \mathrm{C}$ no verão, precipitação média de $1000 \mathrm{~mm}$, com maior frequência de chuva entre os meses de dezembro e março (GOMES et al., 2012), além de belezas naturais. A reunião desses fatores faz com que o número de adeptos à modalidade cresça a cada ano entre seus habitantes, assim como as assessorias esportivas voltadas para a orientação de atividades no mar.

Na praia de Copacabana, mais precisamente no Posto 6, encontra-se atualmente o maior número de assessorias de atividades em águas abertas no Rio de Janeiro. Isto porque a região reúne características como a boa infraestrutura de transporte público, com quatro estações de metrô e mais de oitenta linhas de ônibus; o espaço litorâneo mais abrigado, protegido por uma barreira de corais; o grande número de turistas durante todo o ano; além do grande número de moradores.

Especificamente em relação aos praticantes de NAA com fins recreacionais, além dos objetivos primários que frequentemente são a busca por um melhor estado geral de saúde e qualidade de vida, bem-estar físico e emocional, contato com a natureza, prazer e distração, uma boa parte vê, também, uma possibilidade de aumentar os vínculos interpessoais. Inclusive, para alguns, este torna-se o objetivo primário e o esporte torna-se uma ferramenta para estreitar as relações sociais. (ANDERSEN; OTTESEN; THING, 2019). 
Uma pandemia de um novo tipo de coronavírus, o Sars-CoV-2 que provoca a doença COVID-19, marcou o início do ano de 2020 (WORLD HEALTH ORGANIZATION, 2020a). Por ser muito mais agressivo que os demais tipos de coronavírus em relação à gravidade das complicações respiratórias e mortalidade, além da sua rápida velocidade de transmissão, fácil contágio e o crescente número de pessoas infectadas em todo o mundo (LAUER et al., 2020), a Organização Mundial da Saúde (OMS) declarou estado de emergência global no dia 30 de janeiro. (WORLD HEALTH ORGANIZATION, 2020b).

Entre as medidas propostas pela OMS para conter a propagação da Covid-19, impedindo o aparecimento de novos casos, está incluído o distanciamento social (WORLD HEALTH ORGANIZATION, 2020c). O distanciamento social determinou a proibição, entre outros, da prática de atividades esportivas coletivas e exercícios físicos ao ar livre (WALKER et al., 2020). Esta medida gerou a necessidade do replanejamento do modo de se exercitar, ou seja, do ar livre para dentro de casa, e do contato social pessoal para o contato social virtual. Particularmente em relação à NAA, do mar para o quarto ou a sala. A necessidade de ajuste mostrou-se bilateral: tanto os professores quanto os alunos tiveram que se adaptar à nova rotina.

O distanciamento social associado às incertezas em relação ao presente e ao futuro frente à pandemia da Covid-19, pode trazer repercussões negativas em aspectos físicos, psicológicos, espirituais e emocionais. Em Hong Hong, por exemplo, foi constatada a presença de ansiedade e alto nível de risco percebido na população durante o início da epidemia (KWOK et al., 2020). De forma semelhante, no Iran, constatou-se que o nível de ansiedade era muito alto em $10 \%$ da população, sendo significativamente maior entre mulheres, adultos jovens (21 a 40 anos de idade) e entre aqueles que tinham algum conhecido com o diagnóstico de Covid-19 (MOGHANIBASHI-MANSOURIEH, 2020). Na China, 16,5\% dos participantes de um estudo relataram depressão moderada a severa, $8,1 \%$ estresse moderado a severo, e $28,8 \%$ ansiedade moderada a severa. Essas alterações psicoemocionais mostraram-se relacionadas com uma pior autopercepção do estado de saúde (WANG et al., 2020). A qualidade do sono foi outro aspecto apontado como prejudicado em 18\% da população (HUANG; ZHAO, 2020). Além de sintomas psicoemocionais, ficar em casa diminui o gasto calórico diário e muito frequentemente aumenta a ingestão calórica, resultando no ganho de peso. (REBELOS et al., 2020).

A reunião de todos esses aspectos - isto é, alterações psicoemocionais, físicas e estresse - contribui diretamente para um pior funcionamento do sistema imunológico (RAY; 
GULATI; RAI, 2017). E, paradoxalmente, em tempos de Covid-19 o sistema imunológico deve se manter íntegro na sua totalidade de modo a conferir um sistema de defesa eficaz contra microrganismos, especialmente o Sars-CoV-2.

Assim, todos os esforços que favoreçam o fortalecimento do sistema imunológico devem ser empregados e a manutenção da prática de exercícios físicos é uma delas (SIMPSON et al., 2015). No cenário de distanciamento social onde os exercícios físicos ao ar livre e em ambientes fechados como clubes e academias estão suspensos, os recursos tecnológicos de videoconferência pela internet mostraram-se importantes aliados pois, além de permitirem a transmissão em tempo real do conteúdo por instrutores e professores, permitem também a interação entre os participantes.

Os objetivos do presente estudo foram: (i) investigar o impacto da interrupção da prática de NAA devido ao distanciamento social frente à pandemia da Covid-19, na percepção de bem-estar e estado geral de saúde, e (ii) investigar a percepção sobre a necessidade de adaptação em um curto espaço de tempo a um novo modelo de exercício físico, realizado em ambiente domiciliar e mediado por recursos tecnológicos utilizando a internet.

Acredita-se que a percepção de bem-estar e saúde sejam comprometidas pela interrupção da prática de exercícios físicos ao ar livre; porém, a manutenção do estilo de vida ativo, mesmo que mediado por ferramentas de internet, amenize o impacto do distanciamento social não somente pelos benefícios físicos e psicoemocionais proporcionados pelo exercício físico, como também pelo convívio social virtual. A investigação de tais relações é relevante, pois, uma vez esta hipótese sendo confirmada, este novo modelo, para se manter fisicamente ativo, poderá ser ainda mais estimulado em situações adversas, como é o caso do distanciamento social.

\section{MATERIAIS E MÉTODOS}

Para a realização do presente estudo foram coletados depoimentos de praticantes de NAA de uma assessoria esportiva localizada no Posto 6 da Praia de Copacabana, no Rio de Janeiro.

Os praticantes de NAA ( $n=15)$ apresentavam uma frequência semanal de treinamento de NAA de três vezes por semana, porém, no momento do estudo, devido à recomendação da OMS de distanciamento social e consequente proibição das atividades ao ar livre, estavam 
realizando aulas de ginástica em ambiente domiciliar, por meio de videoaula, orientada pela mesma equipe de professores da assessoria.

As videoaulas eram conduzidas por meio do Zoom Meetings (empresa de capital aberto, Nasdaq); uma ferramenta de internet que permite a realização de videoconferências e áudio, seminários, colaborações e conversas em dispositivos móveis, computadores, telefones e sistemas de salas (ZOOM, 2020), nos mesmos horários das aulas no mar. A cada dia era criado e enviado um link para a entrada na videoaula.

Ao final de uma aula, os praticantes de NAA foram informados quanto à realização e aos objetivos do estudo e foram convidados a participar. Para alcançar os objetivos do estudo as seguintes questões foram elaboradas: (i) "Qual o impacto da interrupção da prática de NAA, devido ao distanciamento social na sua percepção de bem-estar e estado geral de saúde?" e (ii) "Qual a sua percepção sobre a necessidade de adaptação, em um espaço de tempo tão curto, a um novo modelo de exercício físico, em um ambiente fechado, e mediado por recursos tecnológicos, utilizando a internet?".

As questões foram enviadas por e-mail e foi solicitado o envio das respostas em até uma semana. Além disso, também foi enviado um Termo de Consentimento Livre e Esclarecido para a concordância de participação no estudo, em que o anonimato dos participantes era resguardado e garantido.

Os dados foram transcritos, analisados individualmente e resumidos em tabelas de acordo com as informações declaradas pelos participantes.

\section{RESULTADOS}

Um total de nove praticantes $(60 \%$ do total de indivíduos que estavam frequentando as videoaulas) respondeu às questões. O grupo apresentou idade mediana $=55$ anos (mínimo=38 e máximo =64 anos), sendo 6 mulheres.

Na Tabela 1 estão apresentadas partes dos depoimentos de cada participante do estudo relativo à questão "Qual o impacto da interrupção da prática de NAA, devido ao distanciamento social na sua percepção de bem-estar e estado geral de saúde?".

Todos os participantes, independente da idade e sexo, relataram que o distanciamento social impactou negativamente na percepção de bem-estar e estado geral de saúde. Os termos mais frequentes nas respostas foram: qualidade do sono (insônia), ansiedade, humor (alegria) e 
aspectos físico e emocional (mental). A perda do contato com a natureza foi um dos fatores que influenciou tais percepções para quase metade dos participantes.

Tabela 1 - Relato dos participantes do estudo para a questão "Qual o impacto da interrupção da prática de NAA, devido ao distanciamento social na sua percepção de bem-estar e estado geral de saúde?"

\begin{tabular}{|c|c|c|c|}
\hline $\mathbf{N}$ & Sexo & $\begin{array}{l}\text { Idade } \\
\text { (anos) }\end{array}$ & $\begin{array}{l}\text { Questão: Qual o impacto da interrupção da prática de NAA, devido ao distanciamento } \\
\text { social na sua percepção de bem-estar e estado geral de saúde? }\end{array}$ \\
\hline 1 & $F$ & 53 & $\begin{array}{l}\text { "No mar você se expõe fisicamente e emocionalmente, diante de situações sempre } \\
\text { diferentes e inesperadas. Sem isso tudo, tenho insônia, stress, mau humor e ganho de } \\
\text { peso." }\end{array}$ \\
\hline 2 & $F$ & 64 & $\begin{array}{l}\text { "O desafio é o de encontrar formas de manter o tônus muscular, a metabolização de } \\
\text { vitamina } D \text { e cálcio. E, também o equilíbrio emocional diante dos horrores crescentes que } \\
\text { vamos assistir/viver com essa pandemia." }\end{array}$ \\
\hline 3 & $F$ & 61 & $\begin{array}{l}\text { "O impacto é muito grande, pois minha rotina de exercícios foi quebrada e o reflexo nas } \\
\text { capacidades físicas e na qualidade do sono é muito grande. Sem falar na estabilidade } \\
\text { emocional que também sofre com a falta dos exercícios e com a falta do contato com a } \\
\text { natureza." }\end{array}$ \\
\hline 4 & $F$ & 55 & $\begin{array}{l}\text { "Impactou na minha saúde, estou tendo mais insônia. Eu não estou me cansando tanto, } \\
\text { como antes, ao exercitar com a natação. Esse foi o maior impacto." }\end{array}$ \\
\hline 5 & $M$ & 60 & $\begin{array}{l}\text { "Você não pode imaginar a falta física e emocional que a acertada suspensão das aulas } \\
\text { em período de Covid-19 me causou." }\end{array}$ \\
\hline 6 & $M$ & 57 & $\begin{array}{l}\text { "Estar sujeito às variáveis climáticas e às condições do mar é um fator de grande alegria } \\
\text { para mim. Evidentemente, sinto enorme falta disso nesse momento em somos requeridos } \\
\text { a permanecer em casa para evitar a disseminação desenfreada da pandemia do Covid- } \\
\text { 19." }\end{array}$ \\
\hline 7 & $F$ & 40 & $\begin{array}{l}\text { "A ausência da convivência com as pessoas, traz, no meu caso, um forte impacto no bem- } \\
\text { estar e na saúde (principalmente emocionalmente)." }\end{array}$ \\
\hline 8 & $F$ & 42 & $\begin{array}{l}\text { "Acredito também no caráter de prover equilíbrio emocional e mental exercido pelas } \\
\text { águas salgadas do mar. Fiquei } 33 \text { dias sem ir ao mar, e senti que realmente meu humor } \\
\text { não era o mesmo." }\end{array}$ \\
\hline 9 & $M$ & 38 & $\begin{array}{l}\text { "O impacto é grande, tanto na disposição diária, quanto no nível de ansiedade e stress. } \\
\text { Faz falta a calma que proporciona a natação no mar." }\end{array}$ \\
\hline
\end{tabular}

Na Tabela 2 estão apresentadas partes dos depoimentos de cada participante do estudo em relação à questão "Qual a sua percepção sobre a necessidade de adaptação, em um espaço de tempo tão curto, a um novo modelo de exercício físico, em um ambiente fechado e mediado por recursos tecnológicos, utilizando a internet?".

A introdução de uma ferramenta tecnológica virtual para a adaptação das atividades esportivas em ambiente fechado foi vista positivamente pela maioria dos participantes do estudo, mesmo que não substitua os prazeres e outros benefícios da prática ao ar livre. Somente um participante relatou não ter se ajustado à nova rotina de exercícios físicos, porém por questões relativas à logística com as novas demandas da casa e da educação domiciliar do filho. 
O aspecto ressaltado como mais positivo foi a possibilidade de um contato/ convívio social, mesmo que virtual.

Tabela 2 - Relato dos participantes do estudo para a questão "Qual a sua percepção sobre a necessidade de adaptação, em um espaço de tempo tão curto, a um novo modelo de exercício físico, em um ambiente fechado e mediado por recursos tecnológicos, utilizando a internet?".

\begin{tabular}{|c|c|c|c|}
\hline $\mathbf{N}$ & Sexo & $\begin{array}{l}\text { Idade } \\
\text { (anos) }\end{array}$ & $\begin{array}{l}\text { "Qual a sua percepção sobre a necessidade de adaptação, em um espaço de tempo } \\
\text { tão curto, a um novo modelo de exercício físico, em um ambiente fechado e mediado } \\
\text { por recursos tecnológicos, utilizando a internet?" }\end{array}$ \\
\hline 1 & $F$ & 53 & $\begin{array}{l}\text { "Comprei corda para pular e um colchonete. Materiais que vão servir para o momento } \\
\text { pós-reclusão também. Portanto, algumas coisas positivas vão permanecer e agora } \\
\text { consigo entender exatamente o que me faz bem. E sinto que finalmente estamos } \\
\text { usando o celular para algo muito positivo." }\end{array}$ \\
\hline 2 & $F$ & 64 & $\begin{array}{l}\text { "A adaptação, do mar para a minha sala com celular, representa a resiliência e } \\
\text { continuidade e está se dando com alegria e algum estranhamento dos exercícios em } \\
\text { solo duro ao invés de areia mole, particularmente os que pedem pulos/saltos. Mas } \\
\text { estou me adaptando bem." }\end{array}$ \\
\hline 3 & $F$ & 61 & $\begin{array}{l}\text { "Os meios tecnológicos são uma ferramenta importante na mediação e na troca de } \\
\text { experiências, mas não substituem as práticas do esporte em si. No meu ver, o ponto } \\
\text { mais importante é a manutenção da convivência, da recreação e de alguns exercícios } \\
\text { alternativos que podem ser feitos em ambientes pequenos e fechados". }\end{array}$ \\
\hline 4 & $\mathrm{~F}$ & 55 & $\begin{array}{l}\text { "O fato de termos capacidade de fazer uso da tecnologia em prol de suavizar essa } \\
\text { época tão delicada que estamos passando é gratificante. Fico muito feliz e satisfeita } \\
\text { com essa adaptação momentânea com as aulas em vídeo. Isso faz com que, de alguma } \\
\text { forma, nos encontremos para nos exercitar e matar as saudades". }\end{array}$ \\
\hline 5 & $M$ & 60 & $\begin{array}{l}\text { "Tem contribuído imensamente para a melhoria do meu bem-estar, o espaço fechado e } \\
\text { o aparato tecnológico somem quando nos vemos, ouvimos as vozes, damos adeus, } \\
\text { soltamos beijinhos, desabafamos. Completamente diferente de baixar um programa de } \\
\text { exercícios que foi gravado para uma multidão de pessoas espalhadas pelo mundo } \\
\text { seguirem, em frente à tela, autônomos. Nossos encontros atuais, mesmo que mediados } \\
\text { pela tecnologia, são acompanhados de afeto e calor humano. Preenchem uma lacuna } \\
\text { enorme que se formou em cada um de nós nesta quarentena". }\end{array}$ \\
\hline 6 & $M$ & 57 & $\begin{array}{l}\text { "Acho que estamos descobrindo que a tecnologia oferece muitos recursos que, se bem } \\
\text { utilizados, podem trazer grande conforto e bem-estar. Fazer uma prática de yoga } \\
\text { guiada por um guru que está na Califórnia ou seguir um curso de uma língua } \\
\text { estrangeira, ministrado por um professor em outro estado da federação, são coisas } \\
\text { inéditas, algo que se não fazia antes por não se ter necessidade ou mesmo interesse." }\end{array}$ \\
\hline 7 & $F$ & 40 & $\begin{array}{l}\text { "É estranho quando estamos acostumados a exercícios ao ar livre, estar num ambiente } \\
\text { fechado. Mas com esse trabalho em conjunto, a experiência tem sido positiva." }\end{array}$ \\
\hline 8 & $F$ & 42 & $\begin{array}{l}\text { "Como diabética tipo } 1 \text { desde 1998, não tenho como ficar sem fazer exercícios físicos. } \\
\text { Isso faz parte da minha vida, pela saúde física e mental. As aulas regulares pela } \\
\text { internet, no mesmo horário das aulas de natação, colaboram para eu manter uma } \\
\text { rotina, continuar acordar cedo e conseguir trabalhar em casa. Converso com alguns } \\
\text { amigos que estão com dificuldade de manter horários e organizar a rotina de home } \\
\text { office. Acredito que para o grupo que migrou para as aulas on-line isso tem sido mais } \\
\text { fácil." }\end{array}$ \\
\hline 9 & $M$ & 38 & $\begin{array}{l}\text { "Se adaptar ao exercício físico em um curto espaço de tempo via recursos tecnológicos } \\
\text { não foi possível para mim e para minha família. Com a nova rotina de estudos em casa } \\
\text { e sem o apoio da nossa empregada doméstica para fazer as tarefas da casa ficou difícil } \\
\text { criar a rotina de exercícios". }\end{array}$ \\
\hline
\end{tabular}




\section{DISCUSSÃO}

Os principais achados do estudo foram que a interrupção da NAA devido ao distanciamento social frente à Covid-19 repercutiu negativamente na percepção de bem-estar e estado geral de saúde dos praticantes. Entre os aspectos mais impactados, destacou-se a qualidade do sono, ansiedade (mostrando-se mais presente) e humor. Estas mudanças foram atribuídas, entre outros fatores, à perda de contato com a natureza. Entretanto, a introdução de uma ferramenta tecnológica de internet, como tentativa de suprir a interrupção da prática de exercícios físicos, foi vista de forma positiva pelos praticantes, sobretudo no que diz respeito à manutenção do convívio social, mesmo que virtual, essencial em tempos de confinamento.

De fato, o distanciamento social como medida para a contenção da propagação da Covid-19 determinou mudanças significativas no modo de vida de toda a sociedade, em diferentes instâncias: educacional, profissional, pessoal, financeiro, incluindo também, a forma de se exercitar (WORLD HEALTH ORGANIZATION, 2020d). Estes ajustes tiveram que ocorrer de uma forma rápida, quase que instantânea e uma boa parte da população estava despreparada para lidar com tal tipo de situação, tornando-se um grande desafio. Particularmente em relação à prática de exercícios físicos em casa, com poucos recursos em termos de equipamentos, mediado por ferramentas tecnológicas virtuais e muitas vezes atividades diferentes das habituais, tem sido uma experiência inédita para muitos.

O uso de ferramentas tecnológicas virtuais para o compartilhamento de informações e comunicação tem crescido de forma expressiva nos últimos anos em todo o mundo (VILLANTI et al., 2017). O maior número de usuários configura também uma maior abrangência de utilização de tais ferramentas, como é o caso do processo ensino-aprendizagem por instituições de ensino (HALPIN; LOCKWOOD, 2019), serviços (LIN; WANG; HUNG, 2020), compras (ROSE; DHANDAYUDHAM, 2014), entre outros. Em termos de exercícios físicos, um grande número de programas de treinamento tem sido desenvolvido, alguns disponibilizados em aplicativos, com objetivos que vão desde a manutenção da aptidão física (VANDELANOTTE et al., 2018) à reabilitação de indivíduos com determinadas condições de saúde (ALLEN et al., 2018). Em geral, são programas com vídeos demonstrativos e orientações sobre a progressão, porém sem o contato social virtual. Os resultados em geral mostram-se positivos (VANDELANOTTE et al., 2018; ALLEN et al., 2018), porém, para muitos o convívio mesmo que virtual com outros indivíduos é importante, como foi constatado no presente estudo. 
Como declarado por um dos participantes, as videoaulas com ferramentas que permitem a visualização e o contato com outros participantes e instrutor/ professor é "completamente diferente de baixar um programa de exercícios que foi gravado para uma multidão de pessoas espalhadas pelo mundo seguirem, em frente à tela, autônomos. Nossos encontros atuais, mesmo que mediados pela tecnologia, são acompanhados de afeto e calor humano. Preenchem uma lacuna enorme que se formou em cada um de nós nesta quarentena".

Assim, percebemos que este tipo de recurso tecnológico de internet, com interação em tempo real entre os participantes, parece ser efetivo para a realização de exercícios físicos em ambiente domiciliar em situações adversas, como é o caso do distanciamento social. Isto, porque além dos benefícios físicos e psicoemocionais que o exercício físico proporciona, possibilita que os indivíduos se vejam e percebam que todos estão vivendo situações semelhantes, compartilhem angústias e sentimentos e se apoiem mutuamente.

É evidente que as videoaulas de ginástica não suprem por completo todos aspectos positivos que a NAA proporciona na saúde e bem-estar, devido ao significado que esta prática possui para cada indivíduo. Significado este que vai muito mais além do físico, emocional, social e espiritual. Contudo, é um consenso, que em tempos de Covid-19, tem sido uma estratégia positiva para minimizar os efeitos negativos causados pelo distanciamento social.

\section{CONSIDERAÇÕES FINAIS}

A interrupção da prática de NAA, devido à recomendação de distanciamento social para a diminuição do contágio da Covid-19, trouxe repercussões negativas na percepção de saúde e bem-estar, principalmente nos aspectos relacionados à qualidade do sono e ansiedade. Entretanto, as videoaulas de ginástica, por meio de ferramentas tecnológicas de internet, mostraram-se positivas para minimizar tais repercussões, sobretudo por permitirem um convívio social, mesmo que virtual.

\section{REFERÊNCIAS}

ALLEN, K. D. et al. Physical therapy vs internet-based exercise training for patients with knee osteoarthritis: results of a randomized controlled trial. Osteoarthritis and Cartilage, [S. I.], v. 26, n. 3, p. 383-396, 2018. DOI 10.1016/j.joca.2017.12.008. Disponível em: 
https://www.oarsijournal.com/article/S1063-4584(17)31381-X/fulltext. Acesso em: 25 abr. 2020.

ANDERSEN, M. H.; OTTESEN, L.; THING, L. F. The social and psychological health outcomes of team sport participation in adults: an integrative review of research. Scandinavian Journal of Public Health, [S. I.], v. 47, n. 8, p. 832-850, 2019. DOI 10.1177/1403494818791405. Disponível em: https://journals.sagepub.com/doi/10.1177/1403494818791405. Acesso em: 25 abr. 2020.

BALDASSARRE, R. et al. Do the fastest open-water swimmers have a higher speed in middleand long-distance pool swimming events? Journal of Functional Morphology and Kinesiology, [S. I.], v. 4, n. 15, p. 1-9, 2019.

FREITAS FILHO, J. B. Q. et al. Perfil dos praticantes de natação em águas abertas da cidade de Fortaleza - Ceará. Motricidade, [S. I.], v. 14, n. 1, p. 336-340, 2018.

GOMES, A. F. et al. Temporal analysis of the relationship between dengue and meteorological variables in the city of Rio de Janeiro, Brazil, 2001-2009. Cadernos de Saúde Pública, [S. I.], v. 28, n. 11, p. 2189-2197, 2012.

HALPIN, P. A.; LOCKWOOD, M. K. K. The use of twitter and zoom videoconferencing in healthcare professions seminar course benefits students at a commuter college. Advances in Physiology Education, [S. I.], v. 43, n. 2, p. 246-249, 2019. DOI 10.1152/advan.00017.2019. Disponível em: https://journals.physiology.org/doi/full/10.1152/advan.00017.2019. Acesso em: 25 abr. 2020.

HUANG, Y.; ZHAO, N. Mental health burden for the public affected by the COVID-19 outbreak in China: who will be the high-risk group? Psychology, Health and Medicine, [S. I.], v. 1, n. 12, p. 113, 2020. DOI 10.1080/13548506.2020.1754438. Disponível em: https://www.tandfonline.com/doi/full/10.1080/13548506.2020.1754438. Acesso em: 25 abr. 2020.

KWOK, K. O. et al. Community responses during early phase of COVID-19 epidemic, Hong Kong. Emerging Infectious Diseases, [S. I.], v. 26, n. 7, p. 1-13, 2020. DOI 10.3201/eid2607.200500. Disponível em: https://wwwnc.cdc.gov/eid/article/26/7/20-0500_article. Acesso em: 25 abr. 2020.

LAUER, S. A. et al. The incubation period of coronavirus disease 2019 (COVID-19) from publicly reported confirmed cases: estimation and application. Annals of Internal Medicine, [S. I.], v. 172, n. 9, p. 577-582, 2020. DOI 10.7326/M20-0504. Disponível em: https://www.acpjournals.org/doi/10.7326/M20-0504. Acesso em: 27 abr. 2020.

LIN, W. R.; WANG, Y. H.; HUNG, Y. M. Analyzing the factors influencing adoption intention of internet banking: applying DEMATEL-ANP-SEM approach. PLoS One, [S. I.], v. 15, n. 2, p. 1-25. DOI 10.1371/journal.pone.0227852. Disponível em: https://journals.plos.org/plosone/article?id=10.1371/journal. pone.0227852. Acesso em: 27 abr. 2020. 
MOGHANIBASHI-MANSOURIEH, A. et al. Assessing the anxiety level of iranian general population during COVID-19 outbreak. Asian Journal of Psychiatry, [S. I.], v. 51, p. 1-5, 2020. DOI 10.1016/j.ajp.2020.102076. Disponível em: https://www.sciencedirect.com/science/article/pii/S1876201820301878?via\%3Dihub. Acesso em: 25 abr. 2020.

OPEN water at the olympics. Swim England Open Water, [S. I.], 2020. Disponível em: https://www.swimming.org/openwater/open-water-olympics/. Acesso em: 25 abr. 2020.

RAY, A.; GULATI, K.; RAI, N. Stress, anxiety, and immunomodulation: a pharmacological analysis. Vitam Horm Journal, [S. I.], v. 103, p. 1-25, 2017. DOI 10.1016/bs.vh.2016.09.007. Disponível em: https://pubmed.ncbi.nlm.nih.gov/28061967/. Acesso em: 27 abr. 2020.

REBELOS, E. et al. Importance of metabolic health in the era of COVID-19. Metabolism, [S. I.], p. 154247, 2020. DOI 10.1016/j.metabol.2020.154247. Disponível em: https://www.ncbi.nlm.nih.gov/pmc/articles/PMC7194666/. Acesso em: 25 abr. 2020.

ROSE, S.; DHANDAYUDHAM, A. Towards an understanding of Internet-based problem shopping behaviour: the concept of online shopping addiction and its proposed predictors. Journal of Behavioral Addictions, [S. I.], v. 3, n. 2, p. 83-89, 2014. DOI 10.1556/JBA.3.2014.003. Disponível em: https://akjournals.com/view/journals/2006/3/2/article-p83.xml. Acesso em: 27 abr. 2020.

SIMPSON, R. J. et al. Exercise and the regulation of immune functions. In: BOUCHARD, C. Progress in molecular biology and translational science. Burlington: Academic Press, 2015. p. 355-380. v. 135. DOI 10.1016/bs.pmbts.2015.08.001. Disponível em: https://www.sciencedirect.com/science/article/pii/S1877117315001842?via\%3Dihub. Acesso em: 25 abr. 2020.

VANDELANOTTE, C. et al. The effectiveness of a web-based computer-tailored physical activity intervention using fitbit activity trackers: randomized trial. Journal of Medical Internet Research, [S. I.], v. 20, n. 12, p. 1-13, 2018. DOI 10.2196/11321. Disponível em: https://www.jmir.org/2018/12/e11321/. Acesso em: 25 abr. 2020.

VILLANTI, A. C. et al. Social media use and access to digital technology in US young adults in 2016. Journal of Medical Internet Research, [S. I.], v. 19, n. 6, p. 196, 2017. DOI 10.2196/jmir.7303. Disponível em: https://www.jmir.org/2017/6/e196/. Acesso em: 26 abr. 2020.

WALKER, P. G. T. et al. Report 12: the global impact of COVID-19 and strategies for mitigation and suppression. Imperial College London, London, p. 1-18, 2020. DOI 10.25561/77735. Disponível em: https://spiral.imperial.ac.uk:8443/handle/10044/1/77735. Acesso em: 25 abr. 2020.

WANG, C. et al. Immediate psychological responses and associated factors during the initial stage of the 2019 coronavirus disease (covid-19) epidemic among the general population in China. International Journal of Environmental Research Public Health, [S. I.], v. 17, n. 5, 2020. DOI 10.3390/ijerph17051729. Disponível em: https://www.mdpi.com/1660-4601/17/5/1729. Acesso em: 25 abr. 2020. 
WORLD HEALTH ORGANIZATION. Novel coronavirus(2019-nCoV): situation report - 22. [Genebra]: WHO, 2020a. Disponível em:

https://apps.who.int/iris/bitstream/handle/10665/330991/nCoVsitrep11Feb2020eng.pdf?sequence=1\&isAllowed=y. Acesso em: 25 abr. 2020.

WORLD HEALTH ORGANIZATION. Novel coronavirus(2019-nCoV): situation report - 11 . [Genebra]: WHO, 2020b. Disponível em: https://www.who.int/docs/defaultsource/coronaviruse/situation-reports/20200131-sitrep-11-ncov.pdf?sfvrsn=de7c0f7_4. Acesso em: 25 abr. 2020.

WORLD HEALTH ORGANIZATION. Novel coronavirus(2019-nCoV): situation report - 72. [Genebra]: WHO, 2020c. Disponível em: https://www.who.int/docs/defaultsource/coronaviruse/situation-reports/20200401-sitrep-72-covid-19.pdf. Acesso em: 26 abr. 2020.

WORLD HEALTH ORGANIZATION. Novel coronavirus(2019-nCoV): situation report - 51 . [Genebra]: WHO, 2020d. Disponível em: https://www.who.int/docs/defaultsource/coronaviruse/situation-reports/20200311-sitrep-51-covid-19.pdf. Acesso em: 27 abr. 2020.

ZOOM. Zoom: líder no gartner magic quadrant 2019 para soluções de reunião. [S. I.], 2020. Disponível em: https://www.zoom.us/. Acesso em: 26 abr. 2020.

Recebido em 28/04/2020

Aceito em 27/05/2020 